\title{
Theory of Weiss oscillations in the magnetoplasmon spectrum of Dirac electrons in graphene
}

\author{
M. Tahir* \\ Department of Physics, University of Sargodha, Sargodha, Pakistan \\ K. Sabeeh \\ Department of Physics, Quaid-i-Azam University, Islamabad, Pakistan
}

(Date textdate; Received textdate; Revised textdate; Accepted textdate; Published textdate)

\begin{abstract}
We present the collective excitations spectrum (magnetoplasmon spectrum) of Dirac electrons in a weakly modulated single graphene layer in the presence of a uniform magnetic field. We consider electric modulation in one-dimension and the magnetic field applied perpendicular to graphene.We derive analytical results for the intra-Landau band plasmon spectrum within the self-consistentfield approach. We find Weiss oscillations in the magnetoplasmon spectrum which is the primary focus of this work. Results are presented for the intra-Landau band magnetoplasmon spectrum as a function of inverse magnetic field. These results are also compared with those of conventional 2DEG. We have found that the Weiss oscillations in the magnetoplasmon spectrum are larger in amplitude compared to those in conventional 2DEG for the same modulation strength, period of modulation and electron density.
\end{abstract}




\section{INTRODUCTION}

Recent progress in the experimental realization of a single layer of graphene has lead to extensive exploration of electronic properties in this system. Experimental and theoretical studies have shown that the nature of quasiparticles in these two-dimensional systems is very different from those of the conventional two-dimensional electron gas (2DEG) realized in semiconductor heterostructures. Graphene has a honeycomb lattice of carbon atoms. The quasiparticles in graphene have a band structure in which electron and hole bands touch at two points in the Brillouin zone. At these Dirac points the quasiparticles obey the massless Dirac equation. In other words, they behave as massless Dirac fermions leading to a linear dispersion relation $\epsilon_{k}=v k$ ( with the characteristic velocity $v \simeq 10^{6} \mathrm{~m} / \mathrm{s}$ ). This difference in the nature of the quasiparticles in graphene from conventional 2DEG has given rise to a host of new and unusual phenomena such as anamolous quantum Hall effects and a $\pi$ Berry phase [1] [2]. These transport experiments have shown results in agreement with the presence of Dirac fermions. The 2D Dirac-like spectrum was confirmed recently by cyclotron resonance measurements and also by angle resolved photoelectron spectroscopy (ARPES) measurements in monolayer graphene[3]. Recent theoretical work on graphene multilayers has also shown the existance of Dirac electrons with a linear energy spectrum in monolayer graphene[4].

It was found years ago that if conventional 2DEG is subjected to artificially created periodic potentials in the submicrometer range it leads to the appearence of Weiss oscillations in the magnetoresistance. This type of electrical modulation of the $2 \mathrm{D}$ system can be carried out by depositing an array of parallel metallic strips on the surface or through two interfering laser beams [5, 6, 7]. Weiss oscillations were found to be the result of commensurability of the electron cyclotron diameter at the Fermi energy and the period of the electric modulation.

These oscillations were found to be periodic in the inverse magnetic field [5, 6, 7]. It is interesting to study the affect of electrical modulation of graphene layer on the Dirac electrons. Theoretical study of transport properties of Dirac electrons in graphene was recently carried out which showed the appearance of enhanced Weiss oscillations in the magnetoconductivity [8].

One of the important electronic properties of a system are the collective excitations (plasmons). Weiss oscillations in the magnetoplasmon spectrum of conventional 2DEG has 
been the subject of continued interest [9]. Plasmons in graphene were studied as early as in the eighties[10] and more recently [11]. What distinguishes our work from earlier studies of plasmons in graphene is that we consider the effect of electric modulation on the magnetoplasmons which has not been studied before. In the present work, we study the affect of electrical modulation of a graphene monolayer on the magnetoplasmon spectrum within the self-consistent-field approach. We also analyze the dynamic, nonlocal dielectric function of this system and in particular consider the modulation-induced effects.

The present paper is arranged as follows. In section II we give the formulation of the problem. We analytically determine the intra-Landau band plasmon spectrum in section III. In section IV we present the numerical results and discussion. Concluding remarks are made in section $\mathrm{V}$.

\section{FORMULATION}

We consider two-dimensional Dirac electrons in graphene moving in the $\mathrm{x}-\mathrm{y}$-plane. The magnetic field $\left(B_{z}\right)$ is applied along the z-direction perpendicular to the graphene plane. This system is subjected to weak electric modulation along the x-direction. We employ the Landau gauge and write the vector potential as $A=(0, B x, 0)$. The two-dimensional Dirac like Hamiltonian for single electron in the Landau gauge is ( $\hbar=c=1$ in this work) [1, 2, 8]

$$
H_{0}=v \sigma \cdot(-i \nabla+e A)
$$

Here $\sigma=\left\{\sigma_{x}, \sigma_{y}\right\}$ are the Pauli matrices and $v$ characterizes the electron velocity. The complete Hamiltonian of our system is represented as

$$
H=H_{0}+U(x)
$$

where $H_{0}$ is the unmodulated Hamiltonian and $U(x)$ is the one-dimensional periodic modulation potential along the x-direction modelled as

$$
U(x)=V_{0} \operatorname{Cos}(K x)
$$

here $K=2 \pi / a, a$ is the period of modulation and $V_{0}$ is the constant modulation amplitude. The Landau level energy eigenvalues without modulation are given by

$$
\varepsilon(n)=v \sqrt{2 e B|n|}=\omega_{g} \sqrt{|n|}
$$


where $\omega_{g}=v \sqrt{2 e B}$ is the cyclotron frequency of the graphene electrons and $n$ is an integer. The Landau level spectrum for Dirac electrons is significantly different from the spectrum for electrons in conventional 2DEG which is given as $\varepsilon(n)=\omega_{c}(n+1 / 2)$, where $\omega_{c}$ is the cyclotron frequency.

Since we are considering weak modulation such that $V_{0}$ is about an order of magnitude smaller than the Fermi energy $E_{F}$, we can apply standard perturbation theory to determine the first order correction to the unmodulated energy eigenvalues in the presence of modulation with the result

$$
U_{n}=\frac{1}{2} V_{0} \operatorname{Cos}\left(K x_{0}\right) e^{-u / 2}\left[L_{n}(u)+L_{n-1}(u)\right]
$$

where $u=K^{2} l^{2} / 2, x_{0}=l^{2} k_{y}, L_{n}(u)$ are Laguerre polynomials and $l=\sqrt{1 / e B}$ is the magnetic length. Hence the energy eigenvalues in the presence of modulation are

$$
\varepsilon\left(n, x_{0}\right)=\varepsilon(n)+U_{n}=v \sqrt{2 e B|n|}+F_{n} \operatorname{Cos}\left(K x_{0}\right)
$$

with $F_{n}=\frac{1}{2} V_{0} e^{-u / 2}\left[L_{n}(u)+L_{n-1}(u)\right]$. This result has been obtained previously [8]. The degeneracy of the Landau level spectrum of the unmodulated system with respect to $k_{y}$ is lifted in the presence of modulation. The formerly sharp Landau levels broaden into bands. The Landau bandwidths $\sim F_{n}$ oscillates as a function of $n$ since $L_{n}(u)$ are oscillatory functions of the index $n$. Similar features were noted in the Landau level spectrum of conventional electrons in density modulated 2DEG. The difference in the case of density modulated Dirac electrons is firstly that the Landau level spectrum depends on the square root of both the magnetic field $B$ and the Landau band index $n$ as against linear dependence in case of conventional electrons. Secondly, for Dirac electrons the energy eigenvalues in the

presence of modulation given by Eq. (6) contain a term which is a linear combination of Laguerre polynomials with indices $n$ and $n-1$ while for conventional electrons there is only a single term that contains Laguerre polynomial with index $n$. We expect that this modulation induced change in the electronic density of states to influence the dielectric response and the collective excitations of the graphene system.

\section{INTRA-LANDAU-BAND PLASMON SPECTRUM OF GRAPHENE}

The dynamic and static response properties of an electron system are all embodied in the structure of the density-density correlation function. We employ the Ehrenreich-Cohen 
self-consistent-field (SCF) approach[12] to calculate the density-density correlation function. The SCF treatment presented here is by its nature a high density approximation which has been successfully employed in the study of collective excitations in low-dimensional systems both with and without an applied magnetic field. It has been found that SCF predictions of plasmon spectra are in excellent agreement with experimental results. Following the SCF approach, one can obtain the dielectric function as

$$
\epsilon(\bar{q}, \omega)=1-v_{c}(\bar{q}) \Pi(\bar{q}, \omega)
$$

where $v_{c}(\bar{q})=\frac{2 \pi e^{2}}{\kappa \bar{q}}, \bar{q}=\left(q_{x}^{2}+q_{y}^{2}\right)^{1 / 2}, \kappa$ is the background dielectric constant and $\Pi(\bar{q}, \omega)$ is the non-interacting density-density correlation function

$$
\begin{aligned}
\Pi(\bar{q}, \omega) & =\frac{1}{\pi a l^{2}} \sum C_{n n^{\prime}}\left(\frac{\bar{q}^{2}}{2 e B}\right) \int_{0}^{a} d x_{0}\left[f\left(\varepsilon\left(n, x_{0}+x_{0}^{\prime}\right)-f\left(\varepsilon\left(n^{\prime}, x_{0}\right)\right)\right]\right. \\
& \times\left[\varepsilon\left(n, x_{0}+x_{0}^{\prime}\right)-\varepsilon\left(n^{\prime}, x_{0}\right)+\omega+i \eta\right]^{-1}
\end{aligned}
$$

where $x_{0}=l^{2} k_{y}, \quad x_{0}^{\prime}=l^{2} q_{y}, C_{n n^{\prime}}\left(\frac{\bar{q}^{2}}{2 e B}\right)=\left(n_{2} ! / n_{1} !\right)\left(\frac{\bar{q}^{2}}{2 e B}\right)^{n_{1}-n_{2}}\left[L_{n_{2}}^{n_{1}-n_{2}}\right]^{2}$ with $n_{1}=$ $\max \left(n, n^{\prime}\right), n_{2}=\min \left(n, n^{\prime}\right)$ and $L_{n}^{l}(x)$ an associated Laguerre polynomial. In writing the above equation we converted the $k_{y}$-sum into an integral over $x_{0}$ by the following relation: $\frac{1}{A} \sum_{k_{y}} \cdots \rightarrow 2 \frac{1}{2 \pi a l^{2}} \int_{0}^{a} d x_{0} \cdots$. This is a convenient form of $\Pi(\bar{q}, \omega)$ that facilitates writing of the real and imaginary parts of the correlation function.

The real and imaginary parts of the density-density correlation function are the essential ingredients in the theoretical considerations of such diverse problems as high frequency and steady state transport, static and dynamic screening and correlation phenomena. The plasmon modes are determined from the roots of the longitudinal dispersion relation

$$
1-v_{c}(\bar{q}) \operatorname{Re} \Pi(\bar{q}, \omega)=0
$$

along with the condition $\operatorname{Im} \Pi(\bar{q}, \omega)=0$ to ensure long-lived excitations. Employing Eq.(8), Eq.(9) can be expressed as

$$
1=\frac{2 \pi e^{2}}{k \bar{q}} \frac{1}{\pi a l^{2}} \sum_{n, n^{\prime}} C_{n n^{\prime}}\left(\frac{\bar{q}^{2}}{2 e B}\right)\left(I_{1}(\omega)+I_{1}(-\omega)\right),
$$

with

$$
I_{1}(\omega)=P \int_{0}^{a} d x_{0} \frac{f\left(\varepsilon\left(n, x_{0}\right)\right)}{\varepsilon\left(n, x_{0}\right)-\varepsilon\left(n, x_{0}+x_{0}^{\prime}\right)+\omega}
$$


( $P$ denotes principal value integral). The plasmon modes originate from two kinds of electronic transitions: those involving different Landau bands (inter-Landau band plasmons) and those within a single Landau-band (intra-Landau band plasmons).

Inter-Landau band plasmons involve the local 1D magnetoplasma mode and the Bernstein-like plasma resonances [9], all of which involve excitation frequencies greater than the Landau-band separation. On the other hand, intra-Landau band plasmons resonate at frequencies comparable to the bandwidths, and the existence of these modes is due to finite width of the Landau levels. The occurrence of such intra-Landau band plasmons is accompanied by $\mathrm{SdH}$ type of oscillatory behavior in $1 / B$. These $\mathrm{SdH}$ oscillations occur as a result of emptying out of electrons from successive Landau bands when they pass through the Fermi level as the magnetic field is increased. The amplitude of these oscillations is a monotonic function of the magnetic field, when the Landau bandwidth is independent of the band index $n$. In the density modulated case, the Landau bandwidths oscillate as a function of the band index $n$, so it is fair to expect that such oscillating bandwidths would affect the plasmon spectrum of the intra-Landau band type, resulting in new kind of oscillations called Weiss oscillations studied extensively in conventional 2DEG. Here, we study these Weiss oscillations in the magnetoplasmon spectrum in graphene.

To examine the intra-Landau-band plasmon spectrum we consider electronic transitions within a single Landau band. In this case $V_{n} \ll \varepsilon_{n}$, this approximation enables us to expand the argument of the distribution function about $\varepsilon_{n}$ and substituting this expansion into Eq.(11) yields

$$
\begin{aligned}
I_{1}(\omega) & =\frac{1}{\omega} \int_{0}^{a} d x_{0} f\left(\varepsilon_{n}+F_{n} \cos \left(K x_{0}\right)\right) \\
& \times\left(1-\frac{F_{n}}{\omega}\left\{\cos \left(K x_{0}\right)-\cos \left(K\left(x_{0}+x_{0}^{\prime}\right)\right)\right\}\right) .
\end{aligned}
$$

The first integral in the above equation vanishes while we fold the range of integration from 0 to $a / 2$ by multiplying a factor of 2 in the second integral. Hence we can express the intra-Landau band plasmon dispersion relation, from Eqs. $(10,11,12)$ and $\mathrm{C}_{n n^{\prime}}(x) \rightarrow 1$ for 
$n=n^{\prime}$, as

$$
\begin{aligned}
1 & =-\frac{16 e^{2}}{k \bar{q}} \frac{1}{\pi a l^{2} \omega^{2}} \sin ^{2}\left(\frac{\pi}{a} x_{0}^{\prime}\right) \\
& \times \sum_{n} F_{n} \int_{0}^{a / 2} d x_{0} f\left(\varepsilon_{n}+F_{n} \cos \left(K x_{0}\right)\right) \cos \left(K x_{0}\right)
\end{aligned}
$$

This can be written as

$$
\omega^{2}=\frac{16 e^{2}}{k \bar{q}} \frac{1}{\pi a l^{2}} \sin ^{2}\left(\frac{\pi}{a} x_{0}^{\prime}\right) \times \sum_{n}\left|F_{n}\right| A_{n}
$$

here $A_{n}=\frac{a}{2 \pi} \frac{\left|F_{n}\right|}{F_{n}} \sqrt{1-\Delta_{n}^{2}} \theta\left(1-\Delta_{n}\right)$ which results from the analytic evaluation of the Fermi function integral in Eq.(13) that can be carried out at $T=0$. The integral contributes only

for $\Delta_{n}<1$, where $\Delta_{n}$ is given by $\Delta_{n}=\left|\frac{\varepsilon_{F}-\varepsilon_{n}}{F_{n}}\right|$ with $\varepsilon_{F}$ the Fermi energy. Hence the zero temperature intra-Landau-band plasmon dispersion relation reduces to

$$
\omega^{2}=\frac{8 e^{2}}{k \bar{q}} \frac{1}{\pi a l^{2}} \sin ^{2}\left(\frac{\pi}{a} x_{0}^{\prime}\right) \times \sum_{n}\left|F_{n}\right| \sqrt{1-\Delta_{n}^{2}} \theta\left(1-\Delta_{n}\right) .
$$

In general, the inter- and intra-Landau-band modes are coupled for arbitrary magnetic field strength. So long as $\left|F_{n}\right|<v \sqrt{n e B}$, mixing of the inter-and intra- Landau band modes is small. Only the intra-Landau-band mode $(\omega)$ will be excited in the frequency regime $v \sqrt{n e B}>\omega \sim\left|F_{n}\right|$.

\section{NUMERICAL RESULTS AND DISCUSSION}

The intra-Landau-band plasma frequency for Dirac electrons given by equation (15) is shown graphically (solid curve) in Fig.1 as a function of $1 / B$. In the same figure we also display the intra-Landau-band plasma frequency for standard electrons in conventional 2DEG (dashed curve). The following parameters were employed for graphene: $k=2.5$, $n_{D}=3 \times 10^{15} \mathrm{~m}^{-2}, v=2.6 \mathrm{eV} \AA, a=382 \mathrm{~nm}$ and $V_{0}=0.5 \mathrm{meV}$. We also take $q_{x}=0$ and $q_{y}=.01 k_{F}$, with $k_{F}=\left(\pi n_{D}\right)^{1 / 2}$. For conventional 2DEG (a 2DEG at the GaAs-AlGaAs heterojunction) we use the following parameters: $m=.07 m_{e}\left(m_{e}\right.$ is the electron mass), $k=12$ and $n_{D}=3 \times 10^{15} \mathrm{~m}^{-2}$ with the modulation strength and period same as in the graphene system. In Fig.1, the modulation induced oscillations due to the 1D modulation are clearly visible superposed on the sharp SdH-type oscillations. The intra-Landau-band plasmons 
have frequencies comparable to the bandwidth and they occur as a result of broadening of the Landau levels due to the modulation in our system. These type of intra-Landau-band plasmons accompanied by regular oscillatory behavior (in $1 / B$ ) of the $\mathrm{SdH}$ type was first predicted in [13] for tunneling planar superlattice where the overlap of electron wavefunction in adjacent quantum wells provides the mechanism for broadening of Landau levels. The $\mathrm{SdH}$ oscillations occur as a result of emptying out of electrons from successive Landau levels when they pass through the Fermi level as the magnetic field is increased. The amplitude of these oscillations is a monotonic function of the magnetic field when the Landau bandwidth is independent of the band index $n$. In the density modulated case, the Landau bandwidths oscillate as a function of the band index $n$, with the result that in the plasmon spectrum of the intra-Landau band type, there is a new kind of oscillation called Weiss oscillation which is also periodic in $1 / B$ but with a different period and amplitude from the SdH type oscillation.

The origin of these two types of oscillations can also be understood by a closer analytic examination of equation (15). In the regime, $v \sqrt{n e B}>\left|F_{n}\right|$, the unit step function vanishes for all but the highest occupied Landau band, corresponding, say, to the band index $N$. The sum over $n$ is trivial, and plasma frequency is given as $\omega \sim\left|F_{N}\right|^{1 / 2}\left(1-\Delta_{N}^{2}\right)^{1 / 4} \theta(1-$ $\left.\Delta_{N}\right)$. The analytic structure primarily responsible for the $\mathrm{SdH}$ oscillations is the function $\theta\left(1-\Delta_{N}\right.$ ), which jumps periodically from zero (when the Fermi level is above the highest occupied Landau band) to unity (when the Fermi level is contained with in the highest occupied Landau band). On the other hand, Weiss oscillations which represent the periodic modulation of the amplitude of SdH oscillation are largely a consequence of the oscillatory factor $\left|F_{N}\right|^{1 / 2}$.

From the results displayed in Fig.1, we can also make a comparison of the Weiss oscillations in the magnetoplasmon spectrum of Dirac electrons in graphene and standard electrons in conventional 2DEG. For the same modulation strength, period of modulation and electron density we find that the Weiss oscillations in graphene have a larger amplitude compared to conventional 2DEG. This can be attributed to the larger characteristic velocity $\left(v \sim 10^{6} \mathrm{~m} / \mathrm{s}\right)$ of electrons in graphene compared to the Fermi velocity of standard electrons and smaller background dielectric constant $k$ in graphene in contrast to conventional 2DEG.

The SCF theory presented here is expected to be a good quantitative approximation since the dimensionless electron density parameter $r_{s}$ in graphene is a constant $(<1)[11$, 
hence we are in the high density regime. It should also be noted that for electron densities under consideration the effective Fermi temperature $T_{F}=E_{F} / k_{B}$ in graphene is very high ( 1300K for $n_{D} \sim 10^{16} \mathrm{~m}^{-2}$ ), so our $T=0$ theory is expected to be valid at higher temperatures. The only constraint is that the intra-Landau band plasmons are due to electronic transitions within a single Landau band and the existence of these modes is due to finite width of the Landau levels caused by the modulation. Therefore modulation induced

effects will be observable for temperatures where the thermal energy is less than the Landau band width such that $k_{B} T<2\left|F_{n}\right|$. In effect the theory is valid for experimental situations where $k_{B} T$ is smaller than $\omega_{g}, F_{n}$ and $\varepsilon_{F}$.

\section{CONCLUSIONS}

We have determined the intra-Landau band plasma frequency for Dirac electrons in a modulated graphene layer in the presence of a magnetic field employing the SCF approach. We show that the intra-Landau band magnetoplasmon spectrum exhibits the electronic density of states modulation in the form of oscillating magnetoplasmon frequencies (Weiss oscillations). We also compare these results with those of the conventional 2DEG. We have found that the Weiss oscillations in the magnetoplasmon spectrum are larger in amplitude compared to those in conventional 2DEG for the same modulation strength, period of modulation and electron density.

\section{ACKNOWLEDGEMENTS}

One of us (K.S.) would like to acknowledge the support of the Pakistan Science Foundation (PSF) through project No. C-QU/Phys (129). M. T. would like to acknowledge the support of the Pakistan Higher Education Commission (HEC).

*Present address: Department of Physics, Blackett Laboratory, Imperial College London, London SW7 2AZ, United Kingdom.

[1] K. S. Novoselov et al., Nature 438, 197 (2005); Y. Zhang et al., ibid. 438, 201 (2005). 
[2] Y. Zheng and T. Ando, Phy. Rev. B 65, 245420 (2002); V. P. Gusynin and S. G. Sharapov, Phys. Rev. Lett. 95, 146801 (2005); N. M. R. Perez et. al. Phys. Rev. B 73, 125411 (2006); M. I. Katsnelson et.al, Nat. Phys. 2, 620 (2006); K. S. Novoselov et al.,Nat. Phys. 2, 177 (2006).

[3] R. C. Deacon et al., arXiv:0704.0410v3; S. Y. Zhou et al., Nat. Phys. 2, 595 (2006).

[4] B. Partoens and F. M. Peeters, Phys. Rev. B 75, 193402 (2007).

[5] D. Weiss et. al., Europhys. Lett., 8, 179 (1989); R. W. Winkler et. al., Phys. Rev. Lett. 62, 1177 (1989); R. R. Gerhardts et. al., Phys. Rev. Lett. 62, 1173 (1989).

[6] P. Vasilopoulos and F. M. Peeter, Phys. Rev. Lett. 63, 2120 (1989).

[7] F. M. Peeters and P. Vasilopoulos, Phys. Rev. B 46, 4667 (1992).

[8] A. Matulis and F. M. Peeters, Phys. Rev. B 75, 125429 (2007).

[9] H. L. Cui, V. Fessatidis and N. J. M. Horing, Phys. Rev. Lett. 63, 2598 (1989); O. G. Balev, N. Studart, P. Vasilopoulos, Phys. Rev. B 62, 15834 (2000), M.S. Kushwaha, Surface Science Reports 41, 1 (2001) and references therein.

[10] K. W. -K. Shung, Phys. Rev. B 34, 979 (1986).

[11] M. -F. Lin, F. -L. Shyu, J. Phys. Soc. Japan 69, 607 (2000), V. Apalkov, X.-F. Wang and T. Chakraborty, Int. J. Mod. Phys. B 21, 1167 (2007); E. H. Hwang and S. Das Sarma, Phys. Rev. B 75, 205418 (2007); X.-F. Wang and T. Chakraborty, Phys. Rev. B 75, 033408 (2007).

[12] H. Ehrenreich and M. H. Cohen, Phys. Rev. 115, 786 (1959).

[13] W. M. Que and G. Kirczenow, Phys. Rev. Lett. 62, 1687 (1989); W. M. Que and G. Kirczenow, Phys. Rev. B 36, 6596 (1987). 